Wir bemerken dabei, daß $\Pi_{\mu \nu}$ ein echter Tensor ist und $\Pi_{\mu}$ bei Eich-Phasentransformationen richtiges Transformationsverhalten aufweist. Die übliche Behauptung ${ }^{2}$, daß die Berücksichtigung des elektro- magnetischen Feldes zur Aufgabe des Verschwindens der kovarianten Ableitung des metrischen Spinors zwingt, erscheint uns also als nicht unbedingt stichhaltig.

Abschließend sei noch auf die interessante Struktur der iterierten Dirac-Gleichung verwiesen: Die Elimination von $\chi^{\dot{A}}$ aus dem Dirac-System ergibt:

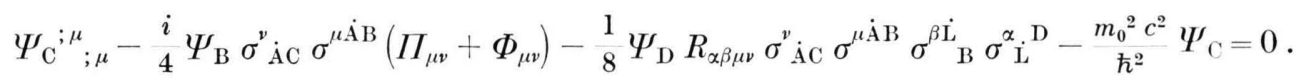

Bei Heranziehung von (25) ergibt sich daraus:

$$
\Psi_{\mathrm{C}}^{; \mu} ; \mu-\frac{i}{4} \Psi_{\mathrm{B}} \sigma_{\dot{\mathrm{AC}}}^{\nu} \sigma^{\mu \dot{\mathrm{AB}}}\left(\Pi_{\mu \nu}+\Phi_{\mu \nu}\right)+\frac{1}{4} R \Psi_{\mathrm{C}}-\frac{m_{0}{ }^{2} c^{2}}{\hbar^{2}} \Psi_{\mathrm{C}}=0 .
$$

Eliminiert man daraus die Krümmungsinvariante $R$ mit Hilfe der Einsteınschen Feldgleichung und beachtet man, daß für die Spur des Energietensors des Dirac-Feldes gilt:

$$
T=2 m_{0} c^{2}\left(\Psi_{\dot{\mathrm{B}}} \chi^{\dot{\mathrm{B}}}+\Psi_{\mathrm{B}} \chi^{\mathrm{B}}\right),
$$

so bekommt man folgende nichtlineare Gleichung ${ }^{2}$ :

$$
\Psi_{\mathrm{C}}^{; \mu} ; \mu-\frac{i e}{2 \hbar c} \sigma_{\dot{\mathrm{A}} \mathrm{C}} \sigma^{\mu \dot{\mathrm{A}} \mathrm{B}} H_{\mu \nu} \Psi_{\mathrm{B}}-\frac{\varkappa}{2} m_{0} c^{2}\left(\Psi_{\dot{\mathrm{B}}} \chi^{\dot{\mathrm{B}}}+\Psi_{\mathrm{B}} \chi^{\mathrm{B}}\right) \Psi_{\mathrm{C}}-\frac{m_{0}{ }^{2} c^{2}}{\hbar^{2}} \Psi_{\mathrm{C}}=0 .
$$

Das kubische Glied dieser Gleichung ist also durch die Gravitationskonstante $\varkappa$ angekoppelt. Eine gewisse Parallele zur Heisenbergschen Materiegleichung ${ }^{6}$ ist einleuchtend, wenn auch die Nichtlinearität hier erst in der iterierten Gleichung über die Gravitation hereinkommt.

Sehen wir uns schließlich noch einmal die Invariante (98) an, so stellen wir fest, daß, sofern

6 H.-P. Dürr, W. Heisenberg, H. Mitter, S. Schlieder u. K. YАMAZAKI, Z. Naturforschg. 14 a, 441 [1959]. der Realteil zur Konstruktion der Lagrange-Funktion für das kombinierte elektromagnetische und gravische Feld brauchbar sein sollte, wir auf folgende Lagrange-Funktion geführt werden:

$$
\Lambda=\frac{\hbar^{2} c^{2}}{32 e^{2}} R_{\lambda \gamma \varrho \varepsilon} R^{\lambda \gamma \varrho \varepsilon}-\frac{1}{4} H_{v \varkappa} H^{\nu \varkappa} .
$$

Untersuchungen über den gravischen Anteil dieser Struktur findet man bei Stephenson und HigGs ${ }^{7}$.

7 G. Stephenson, Nuovo Cim. 9, 263 [1958]. P. W. Higgs, Nuovo Cim. 11, 815 [1959].

\title{
NOTIZEN
}

\section{Thermisches Ausbleichen von Farbzentren in KCl}

Von K. Thommen

Institut für Strahlen- und Kernphysik der Universität Bonn (Z. Naturforschg. 15 a, 362-364 [1960]; eingegangen am 5. März 1960)

Die Verfärbung von Alkalihalogenideinkristallen durch ionisierende Strahlen ist vielfach untersucht worden ${ }^{1}$. Neben der F- und M-Bande entsteht eine Reihe weiterer Banden im Ultravioletten, die teilweise nur bei Temperaturen unterhalb Raumtemperatur stabil sind und als V-Banden bezeichnet werden. Sie werden nach der heutigen Auffassung Fehlstellen zugeschrieben, die aus Kationleerstellen bzw. Aggregaten derselben bestehen, in denen Defektelektronen eingefangen sind. Bei Zimmertemperatur sind von den V-Banden nur die $\mathrm{V}_{2}$-Zentren, bestehend aus zwei assoziierten Kationleerstellen mit zwei eingefangenen Defektelektronen, und die $\mathrm{V}_{3}$-Zentren, bestehend aus zwei assoziierten Kationleerstellen und einem eingefangenen Defektelektron, stabil. Die V-, F- und M-Banden lassen sich unter geeigneten Bedingungen sowohl thermisch als auch optisch ausbleichen. Nach den bestehenden Vorstellungen über die Natur der F-, M- und V-Zentren sollte dabei ein Ausbleichen der F- (und M-) Zentren gleichzeitig ein Ausbleichen der V-Zentren bewirken, da die beim Zerstören der F-(und M-) Zentren freiwerdenden Elektronen mit den Defektelektronen der V-Zentren rekombinieren sollten. DorenDORF $^{2}$ konnte jedoch zeigen, daß es möglich ist, F-Zentren, die durch Bestrahlung mit Röntgen-Strahlen bei

1 F. Seitz, Rev. Mod. Phys. 26, 7 [1954].

2 H. Dorendorf, Z. Phys. 129, 317 [1951]. 
Zimmertemperatur hergestellt worden waren, optisch mit Licht von $\lambda>500 \mathrm{~m} \mu$ auszubleichen, ohne dabei die Konzentration der $\mathrm{V}_{3}$-Zentren wesentlich zu vermindern. Sertz $^{3}$ glaubte, dieses Ergebnis am einfachsten damit deuten zu können, daß ein Teil der Elektronen, die beim Ausbleichen aus den F-Zentren befreit werden, das Innere des Kristalls verläßt und $\mathrm{Na}^{+}$-Ionen an der Oberfläche zu Na-Atomen reduziert, die mit Fremdatomen, etwa Sauerstoff aus der Luft, reagieren. Der Kristall würde nach dieser Interpretation mit einem stöchiometrischen Überschuß an Halogen zurückgelassen werden.

Verfasser ist im Rahmen allgemeinerer Untersuchungen über die Einwirkung energiereicher, geladener Teilchen auf Alkalihalogenideinkristalle zu Ergebnissen gekommen, die in Zusammenhang mit dem oben Aufgeführten mitteilenswert erscheinen.

Ein KCl-Kristall von $5 \times 6 \times 1 \mathrm{~mm}^{3}$ wurde im Synchrozyklotron bei Raumtemperatur mit $\alpha$-Teilchen von etwa $50 \mathrm{MeV}$ Energie bestrahlt. Die Intensität wurde so klein gehalten, daß die Probentemperatur stets unterhalb $45^{\circ} \mathrm{C}$ blieb. Nach der Bestrahlung war der Kristall stark verfärbt (Abb. 1). Das Absorptionsspektrum zeigt die F-Bande, die M-Bande, eine starke Bande im UV mit einem Maximum bei etwa $226 \mathrm{~m} \mu$ und eine sehr schwache Bande bei etwa $340 \mathrm{~m} \mu$. Die starke UV-Bande stellt eine Überlagerung der $\mathrm{V}_{2^{-}}$und $\mathrm{V}_{3}$-Bande dar, die schwache UV-Bande ist wegen ihrer außerordentlich geringen Intensität für das Folgende ohne Bedeutung. Die Absorptionsmessungen wurden mit einem Zeiß-Spektralphotometer bei Zimmertemperatur durchgeführt. Der Kristall wurde anschließend 15 Stunden bei $200^{\circ} \mathrm{C}$ getempert. Abb. 2 zeigt das Absorptionsspektrum nach dem Tempern. Die F- und M-Bande sind völlig ausgebleicht, die $\mathrm{V}_{2^{-}}$und $\mathrm{V}_{3}$-Bande jedoch teilweise erhalten geblieben, wobei sich das Konzentrationsverhältnis $\mathrm{V}_{2}: \mathrm{V}_{3}$--Zentren zugunsten der $\mathrm{V}_{3}$-Zentren verschoben hat. Qualitativ ist dies somit ein Kristall im gleichen Zustand wie der, den Dorendorf durch optisches Ausbleichen hergestellt hatte: Er enthält nur noch V-Zentren. Dieser Kristall wurde nun abermals getempert, diesmal 13 Stunden bei $300^{\circ} \mathrm{C}$. Nach dem Tempern war keine Absorption im Gebiet zwischen 200 und $2500 \mathrm{~m} \mu \mathrm{mehr}$ feststellbar. Auch nach mehreren Tagen war im angegebenen Wellenlängenbereich keine Absorption festzustellen. Es ist daher ausgeschlossen, daß durch das zweite Tempern die verbliebenen V-Zentren in einen metastabilen Zustand befördert wurden, aus dem sie nach gewisser Zeit wieder in den Ausgangszustand zurückkehren, wie dies Alexander und Schneider bei ihren Untersuchungen über das optische Ausbleichen der VZentren beobachtet hatten ${ }^{4}$.

Da schwere geladene Teilchen außer durch Elektronenanregung bzw. Ionisation auch noch durch direkte elastische Stöße auf die Festkörperatome wirken, mußte die Möglichkeit in Betracht gezogen werden, daß das beobachtete Verhalten des Kristalls auf solche spezifische Strahlenschäden schwerer Teilchen zurückzuführen ist. Um dies zu prüfen, wurde ein Kristall bei $6{ }^{\circ} \mathrm{C}$ mit

3 F. Seitz, Rev. Mod. Phys. 26, 62 [1954].

- J. Alexander u. E. E. Schneider, Nature, Lond. 164, 653 [1949].

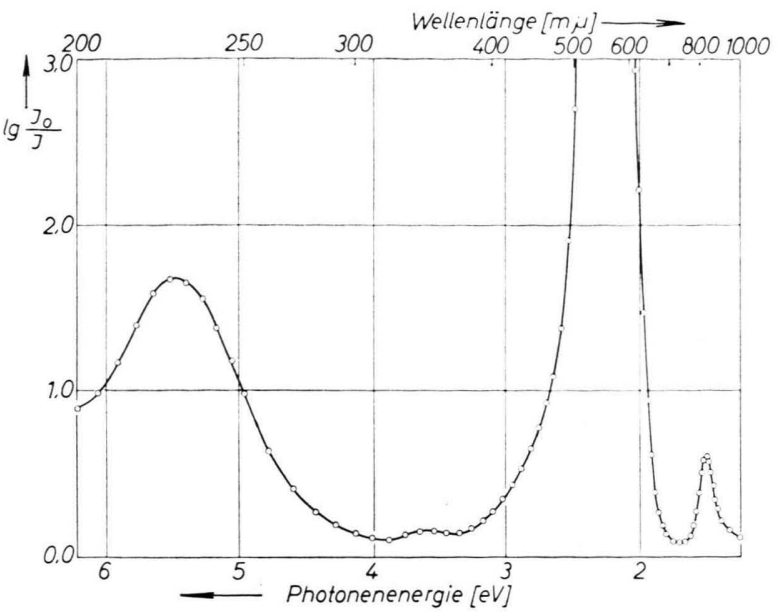

Abb. 1. Absorption eines KCl-Kristalls nach Bestrahlung mit $4 \cdot 10^{13}$ a-Teilchen $/ \mathrm{cm}^{2}$ von etwa $50 \mathrm{MeV}$ Energie.

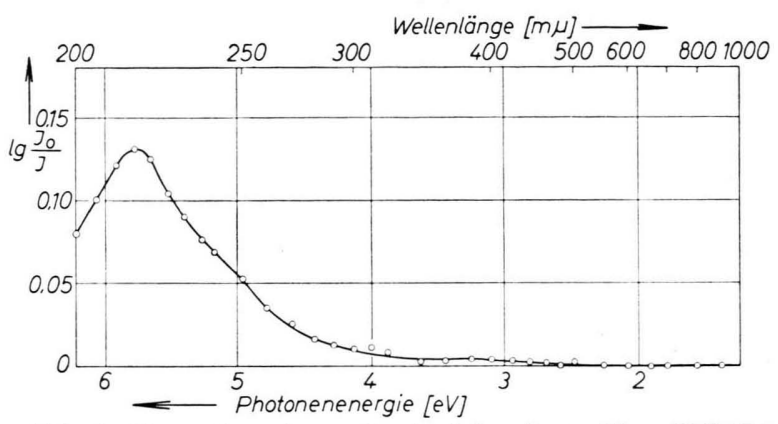

Abb. 2. Absorption eines mit $\alpha$-Teilchen bestrahlten $\mathrm{KCl}-\mathrm{Kri}$ stalls nach 15-stündigem Tempern bei $200^{\circ} \mathrm{C}$.

Röntgen-Strahlen bestrahlt $(70 \mathrm{kV}, 1,6 \mathrm{~mA}$, Wolframantikathode, Bestrahlungsdauer $14 \mathrm{~h}$ ). Das Absorptionsspektrum wurde unmittelbar nach der Bestrahlung, nach 3,5-stündigem Tempern bei $150^{\circ} \mathrm{C}$ und nach 7 -stündigem Tempern bei $150^{\circ} \mathrm{C}$ gemessen. Tab. 1 zeigt die Ergebnisse. Sie sind qualitativ die gleichen wie bei der Bestrahlung mit $\alpha$-Teilchen. Nach 13-stündigem Tempern bei $300{ }^{\circ} \mathrm{C}$ war gleichfalls zwischen 200 und $2500 \mathrm{~m} \mu$ keine Bande mehr zu entdecken. Damit ist gezeigt, daß das beobachtete Verhalten des Kristalls nicht auf spezifische Strahlenschäden schwerer Teilchen zurückzuführen ist.

\begin{tabular}{|c|c|c|c|}
\hline$t[\mathrm{~h}]$ & $E_{\mathrm{F}}$ & $E_{\mathrm{V}}$ & $\lambda_{\mathrm{V}}[\mathrm{m} \mu]$ \\
\hline 0 & 1,50 & 0,34 & 230 \\
3,5 & 0,04 & 0,08 & 215 \\
7,0 & $<0,003$ & 0,05 & 215 \\
\hline
\end{tabular}

Tab. 1. $t$ Temperzeit bei $150{ }^{\circ} \mathrm{C}, E_{\mathrm{F}}$ Absorption $\left(\lg I_{0} / I\right)$ im Maximum der F-Bande, $E_{\mathrm{V}}$ Absorption im Maximum der $\left(\mathrm{V}_{2}+\mathrm{V}_{3}\right)$-Bande, $\lambda_{\mathrm{v}}$ Lage des Maximums der $\left(\mathrm{V}_{2}+\mathrm{V}_{3}\right)$-Bande.

Die hier beschriebenen Versuche führen somit zu dem Ergebnis:

1. Es ist möglich, durch ionisierende Strahlen und ge- 
eignete thermische Behandlung einen KCl-Kristall herzustellen, der nur noch V-Zentren enthält.

2. Diese V-Zentren lassen sich durch weitere thermische Behandlung wieder zerstören.

Will man an dem erwähnten V-Zentren-Modell festhalten, so muß man folgern, daß unter den genannten Versuchsbedingungen die teilweise Erhaltung der VZentren nicht durch eine Abwanderung von Elektronen aus dem Kristallinnern erklärt werden kann. Verfasser kommt daher zu dem Schluß, daß es im Kristall für Elektronen Fallen geben muß, in denen sie durch Licht mit $200<\lambda<2500 \mathrm{~m} \mu$ praktisch nicht angeregt werden können. In diese Fallen wird beim ersten Tempern ein Teil der aus den F-Zentren befreiten Elektronen eingefangen. Beim zweiten Tempern wird auch der Rest der Defektelektronen aus den V-Zentren befreit und rekombiniert mit den in den Fallen festgehaltenen Elektronen.

Es ist zu vermuten, daß auch die von Dorendorf beim optischen Ausbleichen beobachtete teilweise Erhaltung von V-Zentren durch die Existenz solcher „unsichtbaren“ Elektronenfallen zu erklären ist.

\section{Das Szintillationsverhalten einiger anorganischer Leuchtstoffe bei tiefer Temperatur}

\section{Von R. Langkau}

Physikal. Staatsinstitut (I. Institut f. Experimentalphysik), Hamburg

(Z. Naturforschg. 15 a, 364 [1960] ; eingegangen am 26. März 1960)

Es wurde das Fluoreszenzabklingen der durch $\alpha$-Teilchen von $\sim 4 \mathrm{MeV}$ angeregten Szintillationen bei der Temperatur des flüssigen Stickstoffs $\left(-195^{\circ} \mathrm{C}\right)$ an den anorganischen Leuchtstoffen $\mathrm{ZnS}(\mathrm{Ag}), \mathrm{ZnO}$ und $\mathrm{CaWO}_{4}$ untersucht. Dabei zeigten sich erhebliche Abweichungen gegenüber dem Szintillationsverhalten bei Zimmertemperatur.

Die Abklingkurven wurden hierzu aus den Spannungsimpulsen an der Anode eines FS-9A-Photomultipliers durch graphische Differentiation unter Berücksichtigung der Arbeitszeitkonstanten gewonnen. Der Kurvenverlauf läßt sich bei beiden Temperaturen zufriedenstellend deuten unter der Annahme, daß der hier untersuchte Szintillationsvorgang sich aus mehreren (maximal drei) exponentiell abfallenden Komponenten zusammensetzt gemäß der Funktion:

$$
I=A_{1} \cdot e^{-t / \tau_{1}}+A_{2} \cdot e^{-t / \tau_{2}}+A_{3} \cdot e^{-t / \tau_{3}} .
$$

Die Ergebnisse sind in der folgenden Tabelle zusammengefaßt, wobei die Intensitäten der einzelnen Komponenten auf die der ersten bezogen sind $\left(A_{1}=1\right)$ :

\begin{tabular}{|l|l|l|l|l|}
\hline & \multicolumn{2}{|c|}{ Zimmertemperatur } & \multicolumn{2}{c|}{$-195{ }^{\circ} \mathrm{C}$} \\
\hline & $A_{1}=1$ & $\tau_{1} \approx 0,07 \mu \mathrm{sec}$ & $A_{1}=1$ & $\tau_{1} \approx 0,07 \mu \mathrm{sec}$ \\
$\mathrm{ZnS}(\mathrm{Ag})$ & $A_{2}=0,13$ & $\tau_{2}=0,55 \mu \mathrm{sec}$ & $A_{2}=0,13$ & $\tau_{2}=0,53 \mu \mathrm{sec}$ \\
& $A_{3}=0,09$ & $\tau_{3}=3,3 \mu \mathrm{sec}$ & $A_{3}=0,03$ & $\tau_{3}=2,45 \mu \mathrm{sec}$ \\
& $A_{1}=1$ & $\tau_{1} \approx 0,09 \mu \mathrm{sec}$ & $A_{1}=1$ & $\tau_{1} \approx 0,08 \mu \mathrm{sec}$ \\
$\mathrm{ZnO}$ & $A_{2}=0,29$ & $\tau_{2}=0,26 \mu \mathrm{sec}$ & $A_{2}=0,57$ & $\tau_{2}=0,35 \mu \mathrm{sec}$ \\
& $A_{3}=0,07$ & $\tau_{3}=1,03 \mu \mathrm{sec}$ & $A_{3}=0$ & - \\
& $A_{1}=1$ & $\tau_{1}=4,1 \mu \mathrm{sec}$ & $A_{1}=1$ & $\tau_{1}=6,8 \mu \mathrm{sec}$ \\
$\mathrm{CaWO}_{4}$ & $A_{2}=0$ & - & $A_{2}=0$ & - \\
& $A_{3}=0$ & - & $A_{3}=0$ & - \\
\hline
\end{tabular}

Tab. 1.
Die bei Zimmertemperatur gemessenen Werte für das $\mathrm{ZnS}(\mathrm{Ag})$ stimmen mit den von Warncke und NeUert ${ }^{1}$ angegebenen überein. Aus den Werten sieht man, daß der Einfluß der tiefen Temperatur sich besonders stark bei der längsten Fluoreszenzkomponente bemerkbar macht. Abb. 1 zeigt am Beispiel des $\mathrm{ZnS}(\mathrm{Ag})$ deutlich

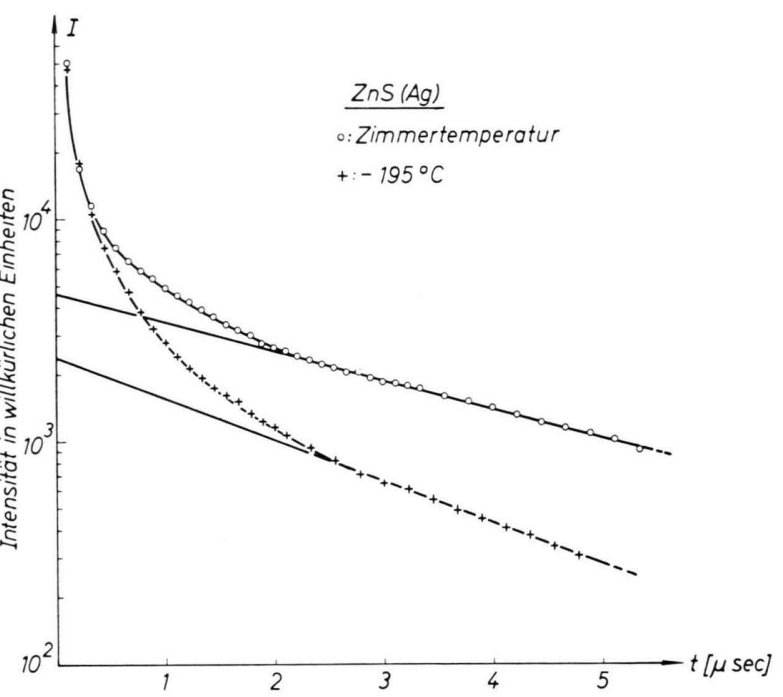

Abb. 1. Szintillationsverlauf bei $\mathrm{ZnS}(\mathrm{Ag})$ für $4-\mathrm{MeV}-\alpha$-Teilchen bei tiefer Temperatur im Vergleich zum Verlauf bei Zimmertemperatur.

den Unterschied im Szintillationsverlauf für die beiden betrachteten Temperaturen. Das Intensitätsverhältnis der dort eingezeichneten langen Komponenten stimmt nicht genau mit den aus der Tabelle folgenden Werten überein, da bei dem gewählten Zeitmaßstab der Abfall im Anfang der Kurve so steil erfolgt, daß ein Anpassen beider Kurven an die gleiche Anfangsintensität nur grob möglich ist. Eine eingehende Beschreibung der Versuche, die auch im Hinblick auf die Möglichkeit der Teilchendiskriminierung durchgeführt werden, soll an anderer Stelle erfolgen.

1 J. W arncke u. H. Nevert, Naturwiss. 47, 11 [1960]. 